Disclosure of Interests: Patricia Bogas: None declared, Chamaida Plasencia: None declared, Francisco Guiñazú: None declared, Marta Novella-Navarro: None declared, Victoria Navarro-Compán Consultant of: Abbvie, Lilly, Novartis, Pfizer, UCB, Speakers bureau: AbbVie, MSD, Lilly, Novartis, Pfizer, UCB, Karen Nathalie Franco Gomez: None declared, Irene Monjo: None declared, Alejandro Balsa Grant/research support from: BMS, Roche, Consultant of: AbbVie, Gilead, Lilly, Pfizer, UCB, Sanofi, Sandoz, Speakers bureau: AbbVie, Lilly, Sanofi, Novartis, Pfizer, UCB, Roche, Nordic, Sandoz DOI: 10.1136/annrheumdis-2020-eular.5862

\section{FRI0087 DURABILITY OF CERTOLIZUMAB PEGOL IN PATIENTS WITH RHEUMATOID ARTHRITIS OR PSORIASIS OVER THREE YEARS: AN ANALYSIS OF POOLED CLINICAL TRIAL DATA}

V. Bykerk ${ }^{1}$, A. B. Gottlieb ${ }^{2}$, K. Reich ${ }^{3}$, Y. Tanaka ${ }^{4}$, K. Winthrop ${ }^{5}$, C. Popova ${ }^{6}$, N. Tilt ${ }^{7}$, A. Blauvelt ${ }^{8} .{ }^{1}$ Hospital for Special Surgery, New York, United States of America; ${ }^{2}$ Icahn School of Medicine at Mount Sinai, New York, United States of America; ${ }^{3}$ University Medical Center Hamburg-Eppendorf, Hamburg, Germany; ${ }^{4}$ University of Occupational and Environmental Health, Kitakyushu, Japan; ${ }^{5}$ Oregon Health and Science University, Portland, United States of America; ${ }^{6}$ UCB Pharma, Brussels, Belgium; ${ }^{7}$ UCB Pharma, Slough, United Kingdom; ${ }^{8}$ Oregon Medical Research Center, Portland, United States of America

Background: Durability over time varies according to the safety, tolerability and efficacy of a drug. ${ }^{1}$ However, durability may vary between patient (pt) subgroups, ${ }^{1,2}$ and physicians should consider pt characteristics when making treatment decisions. Certolizumab pegol (CZP) is an anti-tumour necrosis factor (anti-TNF) agent approved for the treatment of chronic inflammatory diseases, including rheumatoid arthritis (RA) and plaque psoriasis (PSO). ${ }^{3}$ However, little is known about the impact of pt baseline characteristics on long-term CZP durability.

Objectives: To investigate the durability of CZP and reasons for discontinuation over 3 years (yrs) in subgroups of pts with RA or PSO using pooled clinical trial data.

Methods: 27 RA and 3 PSO clinical trials were pooled for indication-specific analyses. Kaplan-Meier curves were calculated to estimate CZP durability for pt subgroups by age, gender, disease duration, prior anti-TNF use and geographic region. Reasons for CZP discontinuation were investigated.

Results: 6927 RA and 1112 PSO pts were included; mean ages were 53.0 yrs (standard deviation [SD]: 12.2 yrs) and 45.4 (13.0) yrs, respectively. 79.3\% RA pts were female (of all patients, $19.4 \%$ were women of childbearing age [18-<45 yrs; WoCBA]) compared with $33.5 \%$ (15.2\% WoCBA) in PSO. Mean disease durations were 6.4 (6.9) yrs for RA and 18.4 (12.3) yrs for PSO. 18.5\% RA and $13.3 \%$ PSO pts had prior anti-TNF use. Maximum CZP exposure was $~ 8$ yrs for

Table 1. CZP durability at 3 years,[a] by patient subgroup

\begin{tabular}{lll}
\hline \% patients & RA & PSO \\
\hline All & 49.2 & 70.1 \\
Age, yrs & & \\
$\quad$ 18-<45 & 52.1 & 66.3 \\
$45-<65$ & 49.4 & 68.3 \\
$\geq 65$ & 43.3 & 69.4 \\
Gender & & \\
$\quad$ Female & 49.3 & 64.1 \\
Male & 48.2 & 69.2 \\
WoCBA & 51.1 & 62.0 \\
Male aged 18-<45 yrs & 56.5 & 68.3 \\
Prior anti-TNF use & & \\
Yes & 49.3 & 60.1 \\
$\quad$ No & 49.6 & 68.5 \\
Disease duration, yrs & & \\
$\quad<1$ & 43.2 & 39.6 \\
1-<5 & 52.6 & 63.6 \\
5-<10 & 51.4 & 64.4 \\
$\geq 10$ & 48.7 & 69.7 \\
Region & & \\
Asia-Pacific & 58.5 & 78.8 \\
Central Europe & 61.5 & \\
Eastern Europe & 54.2 & 53.9 \\
Latin America & 57.1 & 67.7 \\
N America & 36.6 & \\
W Europe & 33.8 & \\
Rest of the world & 66.3 & \\
\end{tabular}

[a] For PSO, the 3 year analysis was calculated with Week 144 data. CZP: certolizumab pegol; N: North; PSO: psoriasis; RA: rheumatoid arthritis; TNF: tumour necrosis factor; W: Western; yrs: years.
RA and $\sim 3$ yrs for PSO. At $1 \mathrm{yr}, 63.4 \%$ of RA pts remained on CZP vs $80.3 \%$ PSO pts, decreasing to $49.2 \%$ RA pts and $70.1 \%$ PSO pts at 3 yrs (Table 1). Reasons for discontinuation, at any time during the trials, included lack of efficacy (RA $13.5 \%$; PSO 1.8\%), adverse events (RA 11.9\%; PSO $8.1 \%$ ), consent withdrawn (RA 6.7\%; PSO 6.7\%), lost to follow-up (RA 1.8\%; PSO 4.3\%), protocol violation (RA 1.7\%; PSO 0.3\%) and other (RA 9.2\%; PSO 8.7\%). In RA pts, CZP durability was lower in the elderly and in pts with disease duration $<1$ yr. In PSO, durability was lower in pts with disease duration $<1$ yr or prior anti-TNF use. Durability was lower in WoCBA pts than male pts aged $18-<45$ yrs for both indications. CZP durability was lower in Western Europe and North America compared to other regions.

Conclusion: Overall, CZP durability was similar to that reported for other antiTNFs with some differences between indication and subgroups. ${ }^{1}$ Factors influencing durability included age, disease duration and geographic region. Gender differences were observed in the 18-45 yrs age group, however, both male and female CZP durability was higher than in older RA pts.

References:

[1] Neovius M. Ann Rheum Dis 2015;74:354-60; 2. Lie E. Ann Rheum Dis 2015;74:970-8; 3. EMA. CIMZIA SmPC 2019. Available at: https://www.ema. europa.eu [Last accessed 09/01/20].

Acknowledgments: This study was funded by UCB Pharma. Editorial services were provided by Costello Medical.

Disclosure of Interests: Vivian Bykerk: None declared, Alice B Gottlieb Grant/ research support from:: Research grants, consultation fees, or speaker honoraria for lectures from: Pfizer, AbbVie, BMS, Lilly, MSD, Novartis, Roche, Sanofi, Sandoz, Nordic, Celltrion and UCB., Consultant of:: Research grants, consultation fees, or speaker honoraria for lectures from: Pfizer, AbbVie, BMS, Lilly, MSD, Novartis, Roche, Sanofi, Sandoz, Nordic, Celltrion and UCB., Speakers bureau:: Research grants, consultation fees, or speaker honoraria for lectures from: Pfizer, AbbVie, BMS, Lilly, MSD, Novartis, Roche, Sanofi, Sandoz, Nor dic, Celltrion and UCB., Kristian Reich Grant/research support from: Affibody; Almirall; Amgen; Biogen; Boehringer Ingelheim; Celgene; Centocor; Covagen; Eli Lilly; Forward Pharma; Fresenius Medical Care; GlaxoSmithKline; Janssen; Kyowa Kirin; LEO Pharma; Medac; Merck; Novartis; Miltenyi Biotec; Ocean Pharma; Pfizer; Regeneron; Samsung Bioepis; Sanofi Genzyme; Takeda; UCB; Valeant and Xenoport., Consultant of: Affibody; Almirall; Amgen; Biogen; Boehringer Ingelheim; Celgene; Centocor; Covagen; Eli Lilly; Forward Pharma; Fresenius Medical Care; GlaxoSmithKline; Janssen; Kyowa Kirin; LEO Pharma; Medac; Merck; Novartis; Miltenyi Biotec; Ocean Pharma; Pfizer; Regeneron; Samsung Bioepis; Sanofi Genzyme; Takeda; UCB; Valeant and Xenoport., Speakers bureau: Affibody; Almirall; Amgen; Biogen; Boehringer Ingelheim; Celgene; Centocor; Covagen; Eli Lilly; Forward Pharma; Fresenius Medical Care; GlaxoSmithKline; Janssen; Kyowa Kirin; LEO Pharma; Medac; Merck; Novartis; Miltenyi Biotec; Ocean Pharma; Pfizer; Regeneron; Samsung Bioepis; Sanofi Genzyme; Takeda; UCB; Valeant and Xenoport., Yoshiya Tanaka Grant/research support from: Asahi-kasei, Astellas, Mitsubishi-Tanabe, Chugai, Takeda, Sanofi, Bristol-Myers, UCB, Daiichi-Sankyo, Eisai, Pfizer, and Ono, Consultant of: Abbvie, Astellas, Bristol-Myers Squibb, Eli Lilly, Pfizer, Speakers bureau: Daiichi-Sankyo, Astellas, Chugai, Eli Lilly, Pfizer, AbbVie, YL Biologics, Bristol-Myers, Takeda, Mitsubishi-Tanabe, Novartis, Eisai, Janssen, Sanofi, UCB, and Teijin, Kevin Winthrop Grant/research support from: Bristol-Myers Squibb, Consultant of: AbbVie, Bristol-Myers Squibb, Eli Lilly, Galapagos, Gilead, GSK, Pfizer Inc, Roche, UCB, Christina Popova Employee of: UCB Pharma, Nicola Tilt Employee of: UCB Pharma, Andrew Blauvelt Consultant of: AbbVie, Aclaris, Almirall, Arena Athenex, Boehringer Ingelheim, Bristol-Myers Squibb, Dermavant, Dermira, Eli Lilly, FLX Bio, Forte, Galderma, Janssen, Leo, Novartis, Ortho, Pfizer, Regeneron, Sandoz, Sanofi Genzyme, Sun Pharma, and UCB Pharma, Speakers bureau: AbbVie

DOI: 10.1136/annrheumdis-2020-eular.1682

\section{FRI0088}

\section{CHANGE IN SERUM BILIRUBIN SUGGESTS} TREATMENT RESPONSE IN RHEUMATOID ARTHRITIS PATIENTS TREATED WITH MOLECULAR-TARGETED AGENTS

S. J. Choi ${ }^{1}$, S. H. Nam ${ }^{1}$, J. S. Lee', W. J. Seo ${ }^{2}$, J. S. Oh ${ }^{3}$, S. $\mathrm{Hong}^{1}$, C. K. Lee ${ }^{1}$, B. Yoo ${ }^{1}$, Y. G. Kim ${ }^{1} .{ }^{1}$ University of Ulsan College of Medicine, Asan Medical Center, Division of Rheumatology, Department of Internal Medicine, Seoul, Korea, Rep. of (South Korea); ${ }^{2}$ Seoul Veterans Hospital, Division of Rheumatology, Department of Internal Medicine, Seoul, Korea, Rep. of (South Korea); ${ }^{3}$ Asan Medical Center, Department of Biomedical Informatics, Seoul, Korea, Rep. of (South Korea)

Background: Bilirubin is an antioxidant with anti-inflammatory properties. In previous reports, serum bilirubin levels were correlated with disease activity of autoimmune diseases including rheumatoid arthritis (RA). Various molecular-targeted 
agents have been developed for RA, and targets, such as IL-6 and TNFa, are associated with liver function. However, the association between serum bilirubin and treatment response in RA patients treated with molecular-targeted agents is still unknown.

Objectives: We aimed to evaluate the role of serum bilirubin in the prediction of the early treatment response in RA patients who initiated molecular-targeted agents.

Methods: We retrospectively recruited biologic naïve RA patients $(n=292)$ with moderate-to-high disease activity from a tertiary hospital between Jan 2013 and Dec 2019. Patients with viral hepatitis, drug-induced hepatitis, or alcoholic liver disease were excluded. Molecular-targeted agents included tocilizumab (TCZ, $n=40$ ), adalimumab (ADA, $n=59$ ), etanercept ( $E T N, n=66)$, golimumab ( $G O L$, $n=60$ ), abatacept (ABA, $n=31$ ), and tofacitinib (TOF, $n=36$ ). Clinical and laboratory data were collected from electronic medical records. Patients were categorised into an increased bilirubin group (higher serum bilirubin at 3 months than at baseline) and decreased bilirubin group (equal or lower serum bilirubin at 3 months than at baseline). At 6 months of treatment, good response (defined as a DAS28 score $\leq 3.2$ ) was evaluated. Multivariate logistic regression analysis and multiple linear regression analysis were used to evaluate the association between serum bilirubin and treatment response. The variables included in the multiple logistic and linear regression analyses were age, female sex, rheumatoid factor, prednisolone, DMARDs, baseline liver enzymes, baseline DAS28 score, and components.

Results: The mean serum bilirubin level at baseline was $4.7 \pm 1.8 \mathrm{mg} / \mathrm{L}$. After 6 months of treatment, $180(61.6 \%)$ patients achieved good responses. The mean serum bilirubin levels at 3 and 6 months were $5.3 \pm 2.3$ and $5.5 \pm 2.2 \mathrm{mg} / \mathrm{L}$, respectively. At 6 months, a good response was more frequent in the increased bilirubin group than in the decreased bilirubin group (71.2\% [99/139] vs. $52.9 \%$ [81/153], $p=0.001)$. In multivariate logistic regression analysis, the ORs among good responders at 6 months were $1.221(95 \% \mathrm{Cl} 1.014-1.471, \mathrm{p}=0.036)$ for baseline serum bilirubin and $1.377(95 \% \mathrm{Cl} 1.146-1.654, \mathrm{p}=0.001)$ for the change in serum bilirubin at 3 months. According to target agents, the mean changes in serum bilirubin from baseline to 6 months were $1.9 \pm 2.5$ for TCZ, $1.0 \pm 1.5$ for $A D A, 0.7 \pm 1.9$ for $E T N, 0.6 \pm 2.2$ for $\mathrm{GOL}, 0.3 \pm 1.2$ for $A B A$, and $0.4 \pm 2.2$ for TOF (Figure 1). Among the target agents, TCZ showed a significant increase in the mean serum bilirubin level at 3 and 6 months from baseline. In multiple linear regression analysis performed on TCZ, the change in bilirubin at 3 months was associated with the DAS28 score at 6 months $(\beta=-0.349$, $\mathrm{p}=0.020)$.
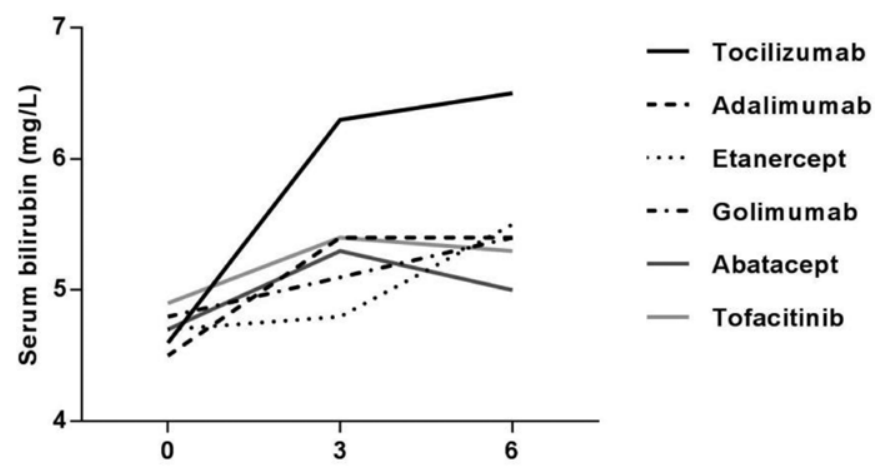

Time from onset of target agents (months)

Figure 1. Change in serum bilirubin during treatment with molecular-targeted agents in rheumatoid arthritis patients

Conclusion: High baseline serum bilirubin and an increase in serum bilirubin during treatment are helpful to predict a good response to molecular-targeted agents, especially TCZ.

Disclosure of Interests: None declared

DOI: 10.1136/annrheumdis-2020-eular.2405

\section{FRI0089 \\ COMPARATIVE EFFICACY (ACR 20) OF TOCILIZUMAB TO OTHER TARGETED IMMUNE MODULATORS (TIM) FOR RHEUMATOID ARTHRITIS: A NETWORK META- ANALYSIS (NMA)}

J. Dang ${ }^{1}$, J. Uyei ${ }^{2}$, Y. Jiang ${ }^{2}$, R. Singh ${ }^{3}$, P. Bilir ${ }^{2}$, A. Karabis ${ }^{4}$, J. Munakata ${ }^{2}$, M. Michalska ${ }^{1}$, J. H. Best ${ }^{1}{ }^{1}$ Genentech, Inc., South San Francisco, United States of America; ${ }^{2} I Q V I A$, San Francisco, United States of America; ${ }^{3} I Q V I A$, Mumbai, India; ${ }^{4}$ IQVIA, Amsterdam, Netherlands
Background: Clinical trial and real-world evidence have both firmly established efficacy and safety of intravenous (IV) and subcutaneous (SC) tocilizumab, both as monotherapy or in combination with a conventional disease modifying antirheumatic drug (cDMARD). However, tocilizumab's relative efficacy to the new Janus kinase (JAK) inhibitor class of therapies is less certain, given the lack of head-to-head trials.

Objectives: To evaluate the relative efficacy of: 1) combination tocilizumab plus CDMARD to other TIMs plus a CDMARD in TIM-naïve or mixed (<20\% TIM-experienced) adults with moderate to severe RA; 2) tocilizumab monotherapy to other TIM monotherapies in TIM-naïve or mixed adults with moderate to severe RA. Efficacy was defined as achievement of an ACR20 response or better at 24 weeks.

Methods: Randomized controlled trials (RCTs) were selected from a recent systematic literature review conducted by the Institute for Clinical and Economic Review (ICER), as well as from a trial for upadacitinib (SELECT-COMPARE, NCT02629159), which was not included in the ICER 2017 report. Treatments included JAK inhibitors (upadacitinib, baricitinib, and tofacitinib), tumor necrosis factor alpha inhibitors (TNFi; adalimumab, certolizumab pegol, etanercept, golimumab, and infliximab), and other non-TNFis (rituximab, sarilumab, tocilizumab, and abatacept). A Bayesian NMA was performed in OpenBUGS and R to evaluate comparative efficacy using a random effects model for combination therapy and fixed effects model for monotherapy. Model selection was based on deviance information criterion. Forest plots of relative risks (RR) are presented.

Results: In combination therapy analysis, a total of 35 studies were included with a pooled study population of 17,508 patients. Study populations were predominantly female (mean $79 \%$, range 39-95\%), and had a baseline mean age of 52 years (range 47-58), mean disease duration of 8 years (range 2-12), and mean DAS28 score of 6 (range 5-7). When compared to cDMARD, all TIMs were 1.69 to 2.22 times more likely to achieve an ACR20 response or better at 24 Weeks (statistically significant) (Figure 1). In pair-wise comparison, tocilizumab IV and SC did not differ from all other TIMs, including JAK inhibitors (no statistically significant difference).

In monotherapy analysis, a total of 5 studies were included with a pooled study population of 1,189 patients. Study populations were predominantly female (mean $82 \%$, range $75-90 \%$ ), had a baseline mean age of 53 years (range $51-54$ ), mean disease duration of 6 years (range 2-9), and mean DAS28 score of 6 (range 5-7). When compared to CDMARD, all TIMs were 1.65 to 1.84 times more likely to achieve ACR20 response or better (statistically significant) (Figure 2). In pair-wise comparison, tocilizumab IV was associated with a greater likelihood of achieving an ACR20 response or better compared to adalimumab ( $R R=1.10$, $95 \%$ credible interval $(\mathrm{Crl})=1.03,1.29)$.

Conclusion: Results of this NMA demonstrate similar efficacy (ACR20 at week 24) between tocilizumab (IV and SC) and other TIMs, including new JAK inhibitors, when used in combination with a cDMARD among TIM-naïve/mixed patient populations. Tocilizumab IV monotherapy had more favorable efficacy than adalimumab monotherapy. Patients unable to tolerate cDMARDs may experience additional value from tocilizumab compared to adalimumab.

Figure 1. ACR20 at 24 weeks (combination therapy) - Compared to cDMARD all TIMs, in combination with CDMARDs, were 1.69 to 2.22 times more likely to achieve an ACR20 response

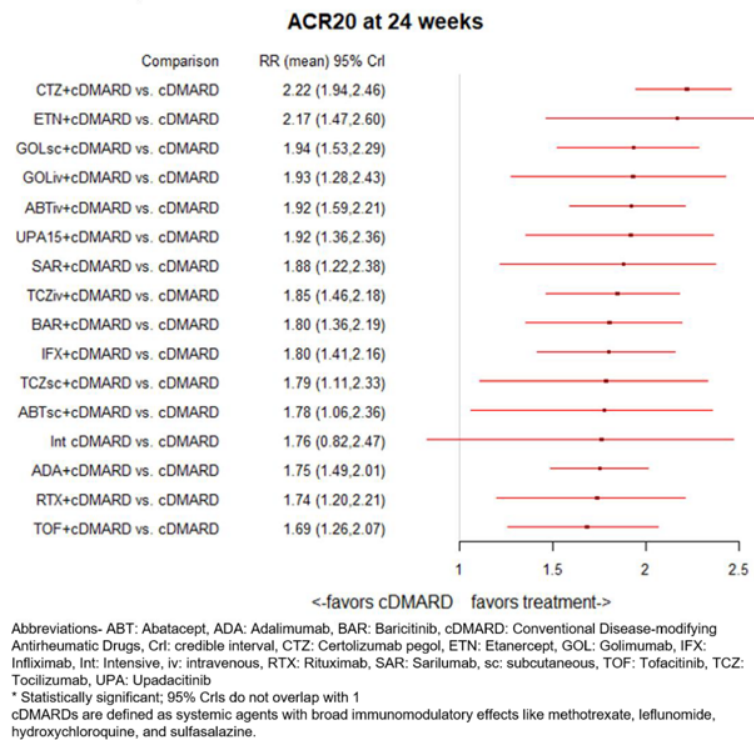

